\title{
Effect of a Transverse Tensile Stress on the Electric-Field-Induced Domain Reorientation in Soft PZT: In Situ XRD Study
}

\author{
Xiaoping Li, ${ }^{*, \dagger}$ Wan Y. Shih, ${ }^{* \dagger}{ }^{\dagger}$ James S. Vartuli, ${ }^{\star}$ David L. Milius, ${ }^{\ddagger}$ \\ Ilhan A. Aksay, ${ }^{* * *}$ and Wei-Heng Shih ${ }^{*, \dagger}$
}

Department of Materials Engineering, Drexel University, Philadelphia, Pennsylvania 19104

Department of Chemical Engineering and Princeton Materials Institute, Princeton University, Princeton, New Jersey 08544-5263

\begin{abstract}
The effect of a transverse tensile stress on the electric-fieldinduced $90^{\circ}$-domain reorientation in tetragonal lead zirconate titanate (PZT) near the morphotropic phase boundary was investigated in situ using X-ray diffraction (XRD). The XRD intensity ratio, $I_{(002)} / I_{(200)}$, which represents the ratio of the volume of the $c$-domains to that of the $a$-domains on the PZT surface, was examined as a function of the electric field at various stress levels. It was found that a transverse tensile stress changes the electric-field dependence of $I_{(002)} / I_{(200)}$, especially at higher electric fields. Without a transverse tensile stress, $I_{(002)} / I_{(200)}$ began to saturate at $E \approx 800 \mathrm{kV} / \mathrm{m}$. With a transverse tensile stress of $75 \mathrm{MPa}, I_{(002)} / I_{(200)}$ increased with an upward curvature with the electric field, indicating that the transverse tensile stress enhanced the field-induced $90^{\circ}$. domain reorientation, and increased the effective piezoelectric coefficients at larger electric fields. At $E=900 \mathrm{kV} / \mathrm{m}$, the estimated $d_{31 \text {,domain }}$ changed from $-200 \times 10^{-12} \mathrm{~V} / \mathrm{m}$ at zero stress, to $-350 \times 10^{-12} \mathrm{~V} / \mathrm{m}$ at $75 \mathrm{MPa}$.
\end{abstract}

\section{Introduction}

$\mathrm{P}^{\prime}$ EZOELECTRIC ceramics for advanced actuator applications often require large displacements under high loadings at large electric fields. Under such conditions, the nonlinear piezoelectric effect in piezoelectric ceramics, such as soft lead zirconate titanate (PZT) occurs. ${ }^{1-6}$ The nonlinear piezoelectric effect in such materials is mainly associated with the domain reorientation processes in the materials, especially the non- $180^{\circ}$-domain reorientation. To better understand and tailor the piezoelectric performance of piezoelectric ceramics under the application conditions at high electric fields, factors that can affect the field-induced domain reorientation process must be investigated. It should be noted that even though soft PZT has a large piezoelectric effect at large electric fields, significant heating could result because of the associated large dielectric loss. Hard PZT ceramics are generally used for high power actuators.

To generate high displacements, there have been several new flextentional actuators developed in recent years, including the Rainbow, ${ }^{7}$ Moonie, ${ }^{8}$ Thunder, ${ }^{9}$ and PrinDrex ${ }^{10,11}$ actuators. A flextentional actuator generally has a piezoelectric coefficient gradient in the thickness direction. When an electric field is applied in the thickness direction, a nonuniform lateral strain

S. E. Trolier-McKinstry-contributing editor

\footnotetext{
Manuscript No. 188724. Received February 24, 2000; approved April 10, 2001 Supported by the Amy Research Office Multidisciplinary University Research Initiative (ARO/MURI) under Grant No. DAAH04-95-1-0102.

${ }^{\star}$ Member, American Ceramic Society.

${ }^{\dagger}$ Drexel University.

Princeton University.
}

distribution developed in the thickness direction causes the actuator to bend, thus producing an axial displacement. The Rainbow actuator consists of a piezoelectric lead lanthanum zirconate titanate (PLZT) plate with one side reduced to a conducting phase. The field-induced axial displacement of a domed Rainbow actuator was $30 \%$ to $40 \%$ larger than that of their flat counterpart. The enhanced axial displacements have been attributed to the residual transverse tensile stress in the PLZT layer originating from the reduction process. ${ }^{12}$ The Thunder actuator consists of a PZT wafer and a thin metal sheet bonded at elevated temperatures using a special polymer adhesive. ${ }^{13}$ The enhanced displacements of a Thunder actuator have also been attributed to the residual transverse tensile stress in the PZT layer caused by the thermalexpansion-coefficient mismatch between the PZT layer and the metal layer. The PrinDrex transducers are laminated cosintered ceramic layers of different piezoelectric coefficients. A domed PrinDrex transducer also exhibited enhanced axial displacements. We speculate that residual transverse tensile stress within the PZT layer was also the probable cause.

Regarding the stress effect on the piezoelectric behavior, there have been several studies on the effect of an axial compressive stress on the piezoelectric coefficients $\left.\left(d_{\mathrm{ij}}\right)\right)^{14-17}$ An axial compressive stress is generally shown to suppress the piezoelectric coefficients. The effect of an axial compressive stress may be important for direct extension applications, where the mass and pressure loading gives the compressive stress in the direction of actuation. However, a lateral stress plays a more important role in flextentional actuators because, with a flextentional transducer, an axial loading gets amplified in the lateral direction. As far as we know, there has been no study about the effect of a transverse stress on the electric-field-induced domain reorientation, especially at higher electric fields. The purpose of this paper is to investigate the effect of a transverse tensile stress on the electricfield-induced non- $180^{\circ}$-domain reorientation in PZT ceramics. The study was conducted on a tetragonal PZT sample with a composition near the morphotropic phase boundary (MPB). The $\mathrm{X}$-ray diffraction (XRD) patterns were obtained in situ by subjecting the samples to an applied electric field at various tensile stress levels. The part of the piezoelectric coefficient that was caused by the domain reorientation, dubbed $d_{31 \text {,domain }}$, was also estimated.

\section{Experimental Procedure}

The PZT samples used for the present study were made from EC-76 PZT powder (Edo Corp., Salt Lake City, Utah) that was predominantly tetragonal with a composition near the MPB. The dry-pressed green bodies were first sintered in an oxygen atmosphere at $1175^{\circ} \mathrm{C}$ for $4 \mathrm{~h}$. The sintered ceramics had an apparent density $>98 \%$ theoretical density. The ceramics were cut into thin rectangular shims of $2.5 \mathrm{~cm} \times 0.5 \mathrm{~cm} \times 0.06 \mathrm{~cm}$. After polishing, a continuous layer of silver paste was deposited on the bottom surface as the bottom electrode, and a thin strip of silver paste on the top surface for soldering, as is schematically shown in Fig. 
1(a). The deposited silver paste was $\sim 1 \mu \mathrm{m}$ thick. After the silver paste was fired at $600^{\circ} \mathrm{C}$ for $40 \mathrm{~min}$, a continuous gold electrode $\sim 200 \AA$ thick was deposited on the top surface to overlap the silver strip. The gold electrode on the top surface was thin enough to allow X-ray penetration. Thin wires were then soldered onto the silver electrode at the bottom and the silver strip on the top for the application of electric fields. Poling was done at $2000 \mathrm{kV} / \mathrm{m}$ in silicon oil at $110^{\circ} \mathrm{C}$ for $30 \mathrm{~min}$.

For poled samples, the XRD studies were conducted two days after the poling. A precision strain gauge (Model EA-06-031DE350, Measurements Group, Inc., Raleigh, North Carolina) was glued to the bottom surface of a sample. The two ends of the sample were then glued to a homemade strain fixture. A schematic of the strain fixture is shown in Fig. 1(b). By turning the finely threaded screw of the strain fixture, a transverse tensile stress was exerted on the sample. Note that the glue that was applied covered the edge, top, and bottom surface of the PZT. As a result, we expect the applied stress on the top and bottom surface to be similar. The strain was directly read through the strain gauge, and the corresponding stress could be obtained from the product of the strain and the Young's modulus. The fixture was designed to fit into the XRD sample holder. The strain gauge was placed at the bottom surface of the samples. It is assumed that the strain measured at the bottom surface is the same for the top surface, where stress is applied. Because the PZT samples were thin, the strain and stress in the thickness direction were assumed to be uniform. Care was taken not to damage the sample surfaces and edges, so as not to initiate cracks in the presence of the applied tensile stress. The electric field was applied in the thickness direction with a 240 A high-voltage power supply (Keithley Instruments Inc., Cleveland, $\mathrm{OH}$ ).

The XRD study was conducted in situ on the top surface of the sample that was covered with the thin gold electrode. The $\mathrm{CuK \alpha}$ radiation was scanned at a rate of $0.6 \% \mathrm{~min}$ in the range containing the (200) and (002) diffraction peaks. Three different transverse tensile stress levels were applied in the experiment, resulting in strain levels of 0,750 , and $1200 \times 10^{-6}$. With a Young's modulus of $62.5 \mathrm{GPa}$ for the PZT samples, these strains correspond to stresses of 0,47 , and $75 \mathrm{MPa}$, respectively. Because the Young's modulus of PZT is nonlinear in the case of appreciable domain switching, these stress levels are approximate values. At each stress level, the XRD pattern was obtained at various electric fields as the electric field was increased from 0 to $900 \mathrm{kV} / \mathrm{m}$, and then decreased to 0. For consistency, at each stress level, the XRD scan

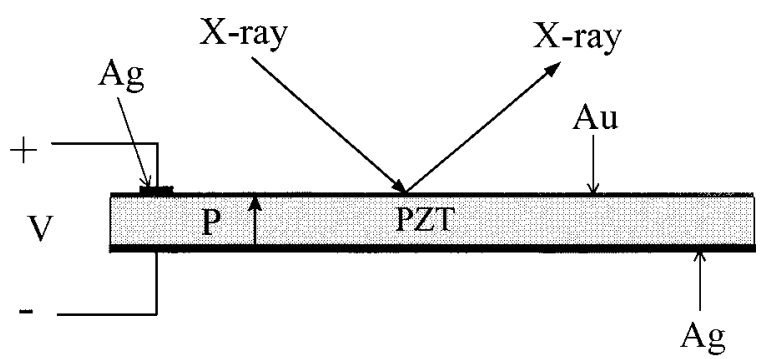

(a)

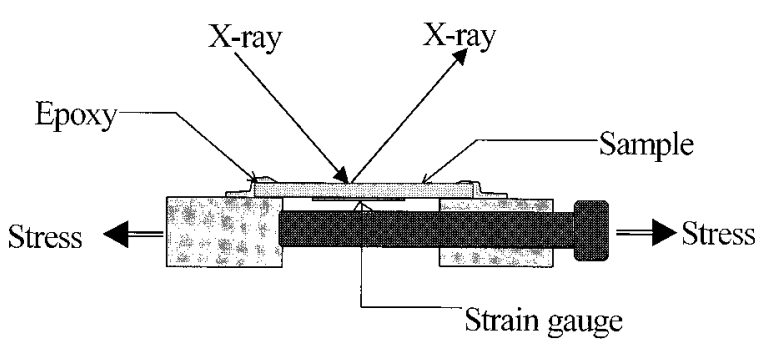

(b)

Fig. 1. A schematic of (a) the PZT sample and the electrodes, and (b) the strain fixture for the in situ XRD study.

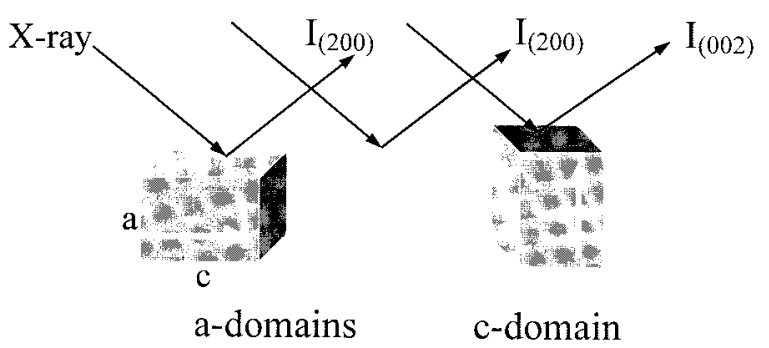

Fig. 2. A schematic of the domain orientations and their XRD. The arrows indicate the polarization direction that is parallel to the c-axis.

began 15 min after the stress was applied. At each electric field, we began the XRD scan 2 min after the electric field was applied. One day of relaxation was allowed between experiments at different stress levels. Because $15 \mathrm{~min}$ is relatively short, the low-field domain structure may be more stabilized than at larger fields. However, these kinetic effects are not considered in the present study.

\section{Results and Discussions}

We refer to domains with their c-axis (polar axis) perpendicular to the XRD planes as c-domains, which were responsible for the (002) diffraction peak. Domains with their c-axis parallel to the XRD planes were referred to as a-domains, which were responsible for the (200) diffraction peak. These domains are schematically illustrated in Fig. 2. The intensities of the (002) and (200) peaks can be correlated with the populations of the c- and a-domains in the sample. From the change in the intensities of the (002) and (200) peaks, one can infer the change in the populations of the cand a-domains, i.e., domain reorientation. Figure 3 shows the (002) and (200) diffraction peaks of an unpoled PZT sample. The intensity of the (002) peak, $I_{(002)}$, and that of the (200) peak, $I_{(200)}$, can be obtained by fitting the diffraction pattern in the vicinity $\sim 20=45^{\circ}$ as a superposition of two Gaussian peaks. An unpoled tetragonal ceramic should have an intensity ratio, $I_{(002)} / I_{(200)}$, of 0.5 if the distribution of the domain orientation is random. As is shown in Fig. 3, the unpoled sample exhibited an $I_{(002)} / I_{(200)}$ greater than 0.5 , indicating a preference of the domains to orient their polarization direction perpendicular to the surface. The c-domain preference on and near the surface was related to the polishing procedure, which has been previously observed in $\mathrm{BaTiO}_{3}, \mathrm{PZT}$, and PLZT. ${ }^{18-21}$ Figure 4 shows the (002) and (200) diffraction peaks of an as-sintered sample (solid line), after being polished with 800 grit $\mathrm{SiC}$ sand paper (broken line) and a $1 \mu \mathrm{m}$

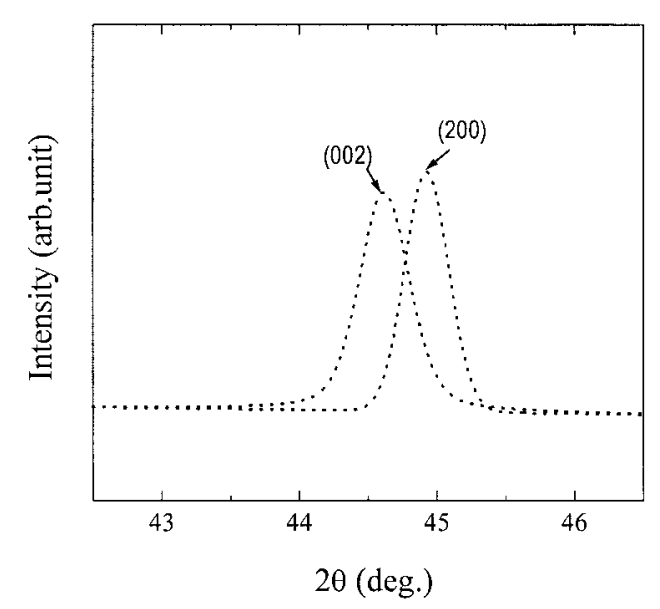

Fig. 3. The (002) and (200) XRD peaks of an unpoled PZT sample after the polishing and electroding procedure. 


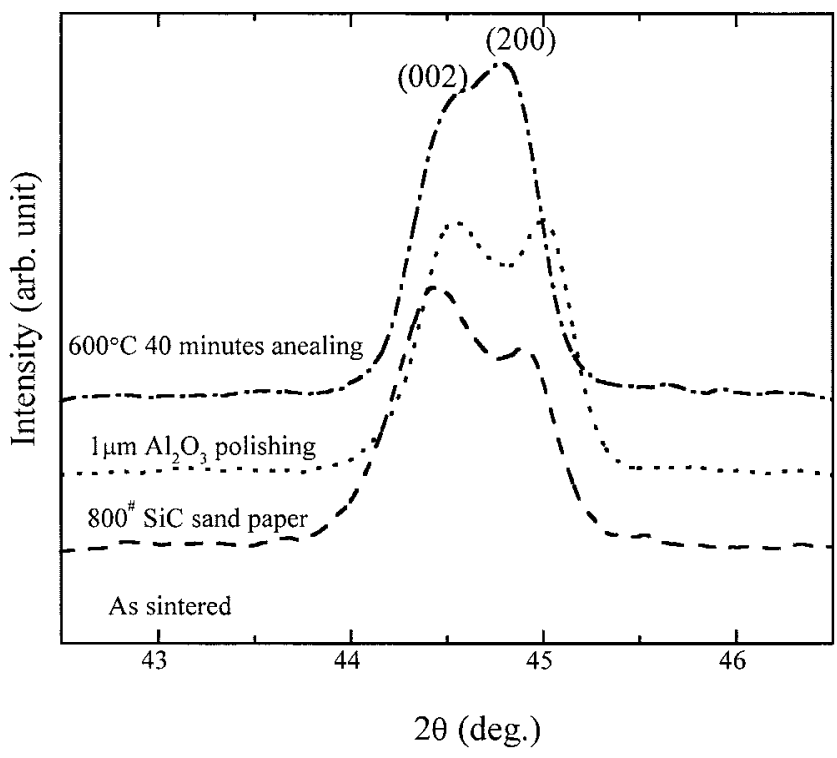

Fig. 4. The XRD peaks of a PZT sample before and after different surface and heat treatments.

$\mathrm{Al}_{2} \mathrm{O}_{3}$ paste (dotted line), and after being heat-treated at $600^{\circ} \mathrm{C}$ for $40 \mathrm{~min}$ following the polishing. As can be seen from Fig. 4, the as-sintered sample had an $I_{(002)} / I_{(200)}$ of $\sim 0.5$. After polishing, the intensity of the (002) peak increased, and that of the (200) peak decreased. The intensity of the (002) peak became about the same as that of the (200) peak when polished with a $1 \mu \mathrm{m} \mathrm{Al}_{2} \mathrm{O}_{3}$ powder, and stronger than that of the (200) peak when polished with 800 grit $\mathrm{SiC}$ sand paper. Because $I_{(\mathrm{OO} 2)} / I_{(200)}$ is larger than 0.5 on the polished surface indicates that the polishing resulted in a c-domain preference on the sample surface. It is not clear how polishing can bring about a c-domain preference. One may speculate that polishing may create defects that contribute to a large internal field near the surface. Although heat treatment at $600^{\circ} \mathrm{C}$ for $40 \mathrm{~min}$ reduced $I_{(\mathrm{OOO})} / I_{(200)}$ to less than unity, it did not completely remove the polishing-induced c-domain preference on the surface (see Fig. 4). As we have illustrated in Fig. 4, $I_{(002)} / I_{(200)}$ was sensitive to the kind of polishing procedure and heat treatment that was given to the sample prior to the XRD measurement. A different polishing procedure and/or heat treatment can result in a different $I_{(002)} / I_{(200)}$. For consistency, all samples received the same polishing procedure, i.e., $1 \mu \mathrm{m} \mathrm{Al}_{2} \mathrm{O}_{3}$ paste polishing, before the electrodes were deposited, followed by the same heat treatment at $600^{\circ} \mathrm{C}$ for $40 \mathrm{~min}$ after the silver paste was deposited.

The effect of a transverse tensile stress on the domain orientation of a poled sample in the absence of an electric field is illustrated in Fig. 5. The (002) and (200) diffraction peaks of the poled PZT sample at zero electric field are shown at a transverse tensile stress of 0,47 , and $75 \mathrm{MPa}$. As can be seen, the intensity of the (200) peak increases, and that of the (002) peak decreases with an increasing transverse tensile stress. $I_{(002)} / I_{(200)}$ decreases from 2.43 at $\sigma=0 \mathrm{MPa}$ to 2.24 at $\sigma=47 \mathrm{MPa}$ and 2.08 at $\sigma=$ $75 \mathrm{MPa}$, where $\sigma$ denotes the transverse tensile stress. This illustrates that a transverse tensile stress favors the a-domain orientation and induces some of the c-domains to switch to a-domains.

The diffraction patterns of the (002) and (200) peaks of a poled sample at various electric fields with and without a transverse tensile stress of $75 \mathrm{MPa}$ are shown in Fig. 6. It can be seen in both sets of curves that the intensity of the (002) peak increased and the intensity of the (200) peak decreased as the electric field was increased. $I_{(002)} / I_{(200)}$ versus the electric field was plotted for all three transverse tensile stresses, i.e., 0, 47, and $75 \mathrm{MPa}$ in Fig. 7. Figure 7 shows that the effect of a transverse tensile stress is threefold:

(1) A transverse tensile stress decreased $I_{(002)} / I_{(200)}$ at zero electric field. $I_{(002)} / I_{(200)}$ decreased from 2.43 at $0 \mathrm{MPa}$ to 2.24 at

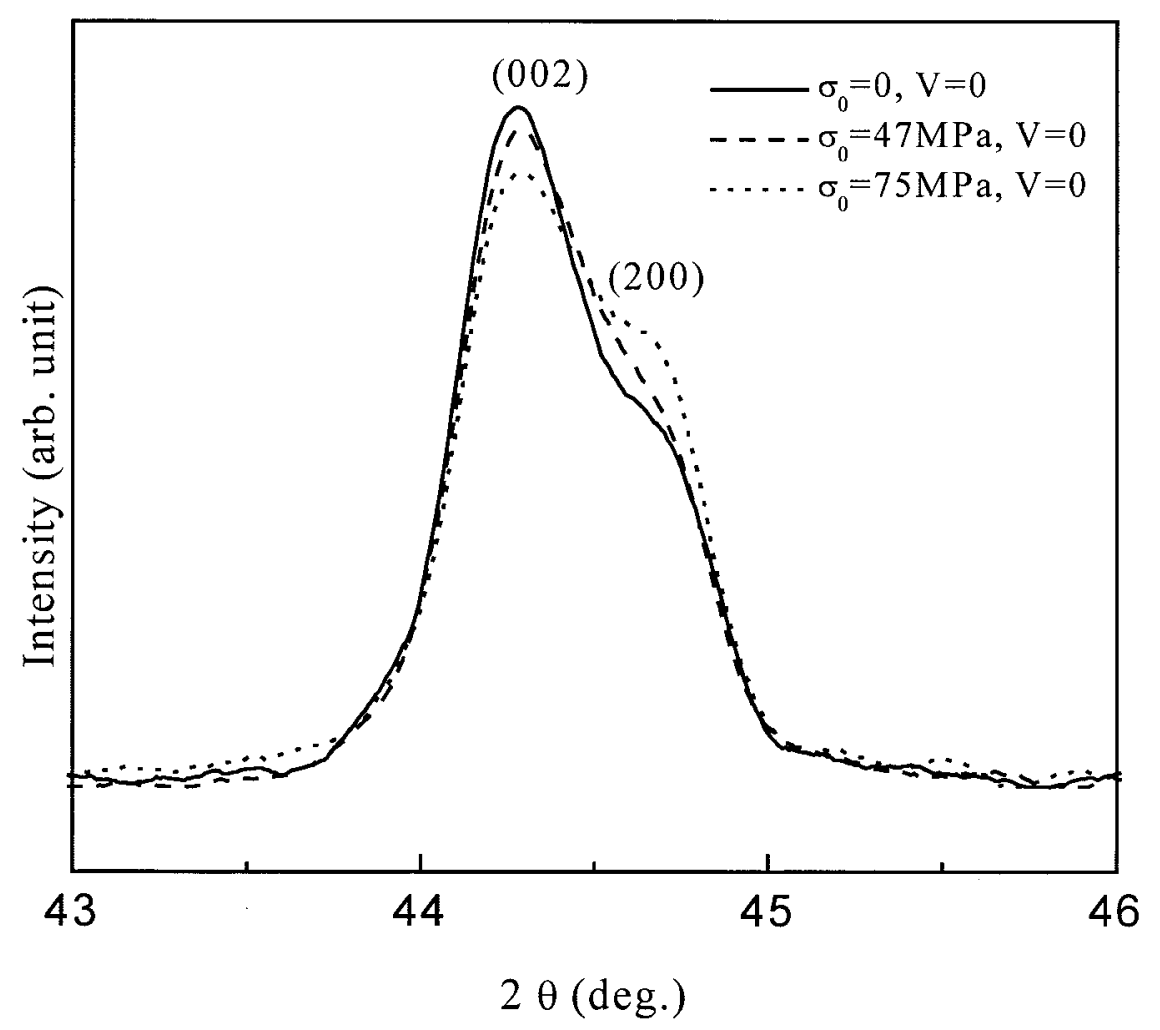

Fig. 5. The zero-electric-field (002) and (200) XRD diffraction peaks of a PZT sample at various transverse tensile stresses. 


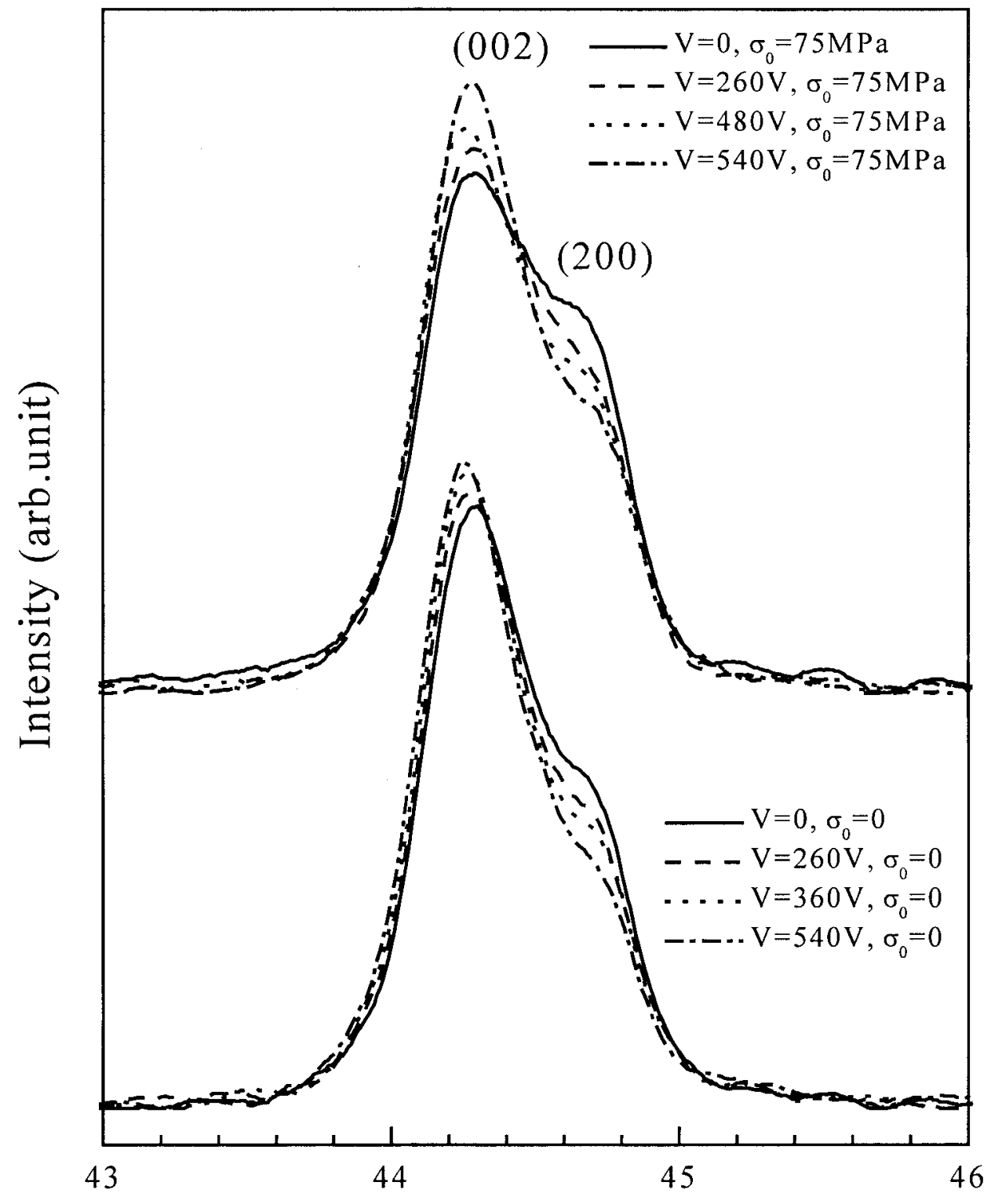

\section{$2 \theta$ (deg.)}

Fig. 6. The (002) and (200) XRD diffraction peaks of a PZT sample at various electric fields with a transverse tensile stress of 0 and $75 \mathrm{MPa}$.

$47 \mathrm{MPa}$ and 2.08 at $75 \mathrm{MPa}$. This indicates that a transverse tensile stress favors a-domain orientation as we have also discussed in Fig. 5.

(2) The presence of a transverse tensile stress changed the way the intensity ratio increased with the electric field. With a zero transverse tensile stress, the intensity increased progressively more slowly as the electric field was increased. The intensity ratio eventually saturated at $\sim 3.0$ at $E=800 \mathrm{kV} / \mathrm{m}$, where $E$ denotes the electric field. In contrast, with a transverse tensile stress, the intensity ratio increased with an increasing electric field without saturation up to $E=900 \mathrm{kV} / \mathrm{m}$. The intensity especially increased with the electric field with an upward curvature, indicating that the presence of a transverse tensile stress enhanced the switching of $\mathbf{a}$-domains to $\mathbf{c}$-domains at higher electric fields. Because of the enhanced domain reorientation by the transverse tensile stress, $I_{(002)} / I_{(200)}$ reached the same value, $\sim 3.0$, at $E=900 \mathrm{kV} / \mathrm{m}$ for all three stress levels, even though the initial $I_{(002)} / I_{(200)}$ at $E=0$ was lower with a transverse tensile stress. The enhanced increase in $I_{(002)} / I_{(200)}$ at higher electric fields by a transverse tensile stress suggested that piezoelectric coefficients could also be enhanced by the transverse tensile stress at higher electric fields.

(3) A transverse tensile stress reduced the degree of irreversibility. As can be seen from Fig. 7, the domain reorientation process was not entirely reversible. This irreversibility created the hysteresis loops in the intensity ratio versus electric field plots shown in Fig. 7. For example, after the electric field was increased to $900 \mathrm{kV} / \mathrm{m}$ and then reduced to zero, the resultant intensity ratio at zero electric field was different from the initial value. The difference between the zero-field intensity ratio after and before one cycle of electric-field sweep, dubbed $\Delta R$, may be used as a measure for irreversibility. From Fig. 7, one can see that $\Delta R$ decreased from 0.4 at $\sigma=0$ to 0.25 at $\sigma=47 \mathrm{MPa}$ and 0.21 at $\sigma=$ $75 \mathrm{MPa}$, indicating that the degree of irreversibility decreases with an increasing transverse tensile stress. The degree of irreversibility may also be caused by the difference in aging time. The long aging time in 


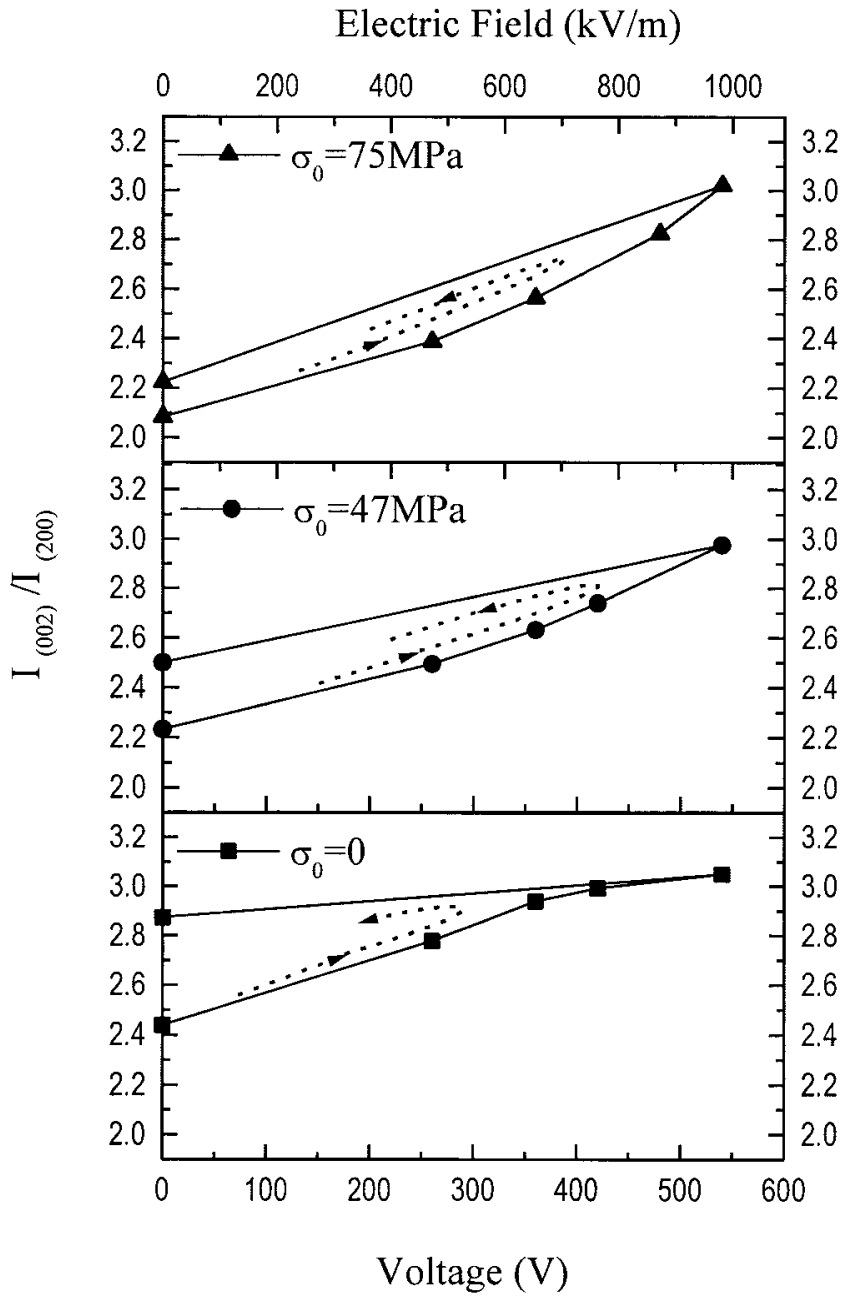

Fig. 7. The $I_{(\mathrm{O02})} / I_{(200)}$ intensity ratio as a function of electric field with various transverse tensile stresses.

the case of $\sigma=0$ resulted in a large $\Delta R$, while a short aging time in the stressed samples resulted in a smaller $\Delta R$.

We speculate that both the enhancement of the electric-fieldinduced domain reorientation and the decrease of the irreversibility in the presence of a transverse tensile stress can be related to the a-domain preference induced by the transverse tensile stress. As we have shown above, in the absence of an electric field, a transverse tensile stress favored the a-domains. Therefore, in the presence of a transverse tensile stress, there would be more a-domains available for reorientation, hence, more a-domains would be reoriented to c-domains when an electric field was applied, resulting in the observed enhancement in the electricfield-induced domain reorientation. Similarly, when the electric field was turned off, the preference for a-domains at zero electric field by a transverse tensile stress caused more c-domains to reorient to a-domains, resulting in reduced irreversibility.

The volume fraction of the electric-field-reoriented domains, $\kappa$, can be quantified by relating $\kappa$ to the change of intensity ratio. ${ }^{20}$ We denote the number of unit cells that belong to a-domains at zero electric field as $A$, and the number of unit cells that belong to c-domains at zero electric field as $C$. The intensity ratio at zero electric field, denoted as $R(0)$, can be related to $C$ and $A$ as

$$
R(0)=\frac{I_{(002)}(0)}{I_{(200)}(0)}=\frac{C}{A}
$$

where 0 denotes zero electric field. When an electric field $E$ is applied, a fraction, $n$, of the a-domains was reoriented into c-domains. The intensity ratio, $R(E)$, can be related to $n, C$, and $A$ as

$$
R(E)=\frac{I_{(002)}(E)}{I_{(200)}(E)}=\frac{n A+C}{(1-n) A}
$$

Combining Eqs. (1) and (2), $n$ can be expressed in terms of $R(0)$ and $R(E)$ as

$$
n=\frac{R(E)-R(0)}{1+R(E)}
$$

The volume fraction, $\kappa$, of the domains that are reoriented by the electric field can be expressed in terms of $R(E)$ and $R(0)$ as

$$
\kappa=\frac{n A}{A+C}=\frac{R(E)-R(0)}{(1+R(E))(1+R(0))}
$$

Using Eq. (4), $\kappa$ can be calculated from the intensity ratio shown in Fig. 7. The resultant $\kappa$ is plotted as a function of the applied electric field in Fig. 8 for transverse tensile stresses of 0, 47, and $75 \mathrm{MPa}$. At zero transverse tensile stress, the volume fraction, $\kappa$, of the reoriented domains increased progressively with an increasing electric field and leveled off at $4 \mathrm{vol} \%$ at $E=900 \mathrm{kV} / \mathrm{m}$. The saturation of $\kappa$ at higher electric fields with zero transverse tensile stress is reminiscent of the saturation of the zero-stress intensity ratio at higher electric fields. At $47 \mathrm{MPa}$, $\kappa$ continued to increase with an increasing electric field to $\sim 6 \%$ at $900 \mathrm{kV} / \mathrm{m}$. At $75 \mathrm{MPa}$, $\kappa$ increased with the electric field with an upward curvature $\kappa$, and reached $8 \%$ at $E=900 \mathrm{kV} / \mathrm{m}$, about twice that at zero stress.

With a tetragonal crystalline structure, $90^{\circ}$-domain reorientation not only changes the intensities of the (200) and (002) peaks, but also the dimension of the specimen. Knowing the type of domain reorientation, it is possible to quantify the part of the piezoelectric coefficient, $d_{31}$, that is caused by domain reorientation. This can be done by relating the volume fraction, $\kappa$, of the reoriented domains to the strain induced by the electric field. In the following, we estimate the $90^{\circ}$-domain reorientation contribution to the $d_{31}$ value. When the electric field $E$ is in the same direction as the polarization as in the present experiment, the strain $\varepsilon_{\text {domain }}(E)$ in the lateral direction, $\mathbf{x}$, caused by the $90^{\circ}$-domain reorientation by the electric field $E$, can be expressed as

$$
\varepsilon_{\text {domain }}(E)=\frac{L(E)-L(0)}{L(0)}
$$

where $L(E)$ and $L(0)$ denotes the length of the specimen with and without the electric field $E$ in the $\mathbf{x}$-direction. In Eq. (5), the intrinsic contribution of the piezoelectric effect within the domains has been neglected. Denoting $\eta(0)$ as the volume fraction of the a-domains that have their $\mathbf{c}$-axis lying in the $\mathbf{x}$-direction at zero electric field, the length of the specimen in the $\mathbf{x}$-direction may be approximated as

$$
L(0)=[\eta(0) c+(1-\eta(0)) a] A+a C
$$

where $c$ and $a$ are the lattice constants of the unit cell in the c-and a-axis, respectively, and $(1-\eta)$ is the volume fraction of the a-domains whose $\mathbf{c}$-axis was perpendicular to the $\mathbf{x}$-direction. With an applied electric field $E$, the length of the specimen in the $\mathbf{x}$-direction may be approximated as

$$
L(E)=[\eta(E) c+(1-\eta(E)) a](1-n) A+a(C+n A)
$$

where $\eta(E)$ and $(1-\eta(E))$ are, respectively, the volume fraction of the a-domains whose $\mathbf{c}$-axis is parallel and perpendicular to the $\mathbf{x}$-direction. In Eqs. (6) and (7), we assume all the domains are either c- or a-domains. This simplification allows us to directly relate the $d_{31}$ coefficient to the volume fraction, $\kappa$, of the reoriented domains. As we will show, the estimated piezoelectric coefficient at $400 \mathrm{kV} / \mathrm{m}$ agreed with that obtained from the resonance method, indicating that the approximation was reasonable. Because the specimen was isotropic in the lateral direction, 


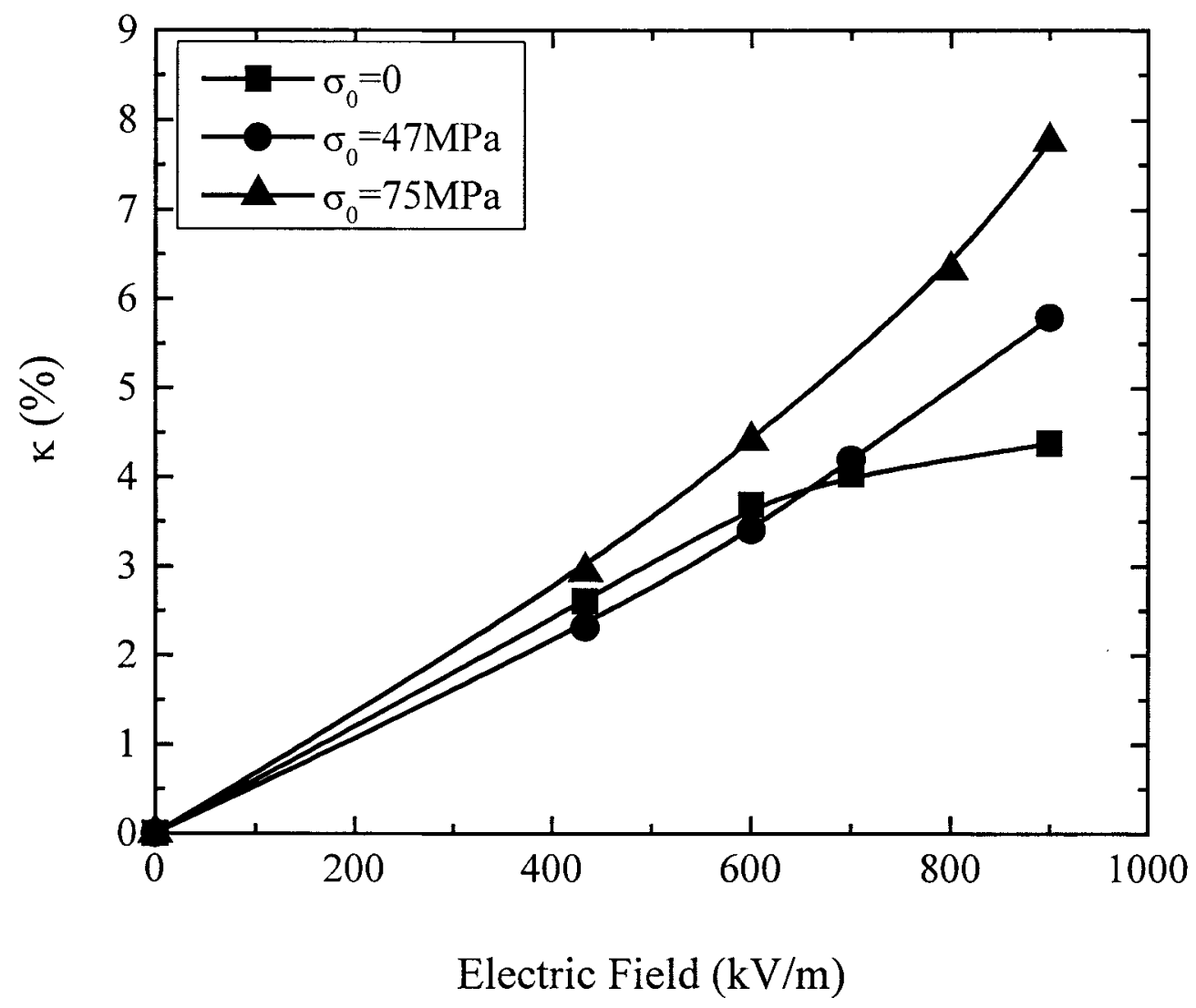

Fig. 8. The volume fraction of the reoriented domains versus electric field at various transverse tensile stresses.

we assume $\eta(0)=\eta(E)=1 / 2$. Substituting Eqs. (6) and (7) into Eq. (5), $\varepsilon$ can be expressed as

$$
\begin{aligned}
\varepsilon(E)=\frac{(c-a)[R(0)-R(E)]}{(1+R(E))[c+a+2 a R(0)]} & \\
= & \frac{\left(1-\frac{c}{a}\right)(1+R(0)) \kappa}{\left(\frac{c}{a}+1+2 R(0)\right)}
\end{aligned}
$$

The part of $d_{31}$ that is caused by the $90^{\circ}$-domain reorientation can be estimated as

$$
d_{31, \text { domain }}=\frac{\varepsilon(E)}{E}=\frac{\left(1-\frac{c}{a}\right)(1+R(0))}{\left(\frac{c}{a}+1+2 R(0)\right)}\left(\begin{array}{l}
\kappa \\
E
\end{array}\right)
$$

where $d_{31 \text {,domain }}$ denotes the part of $d_{31}$ that is caused by domain reorientation. As can be seen from Eq. (9), $d_{31 \text {,domain }}$ is directly proportional to $\kappa$ because $c, a$, and $R(0)$ are constants. Using Eq. (9) with $c / a=1.0084$, as obtained from the XRD data, and $R(0)$ values, as shown in Fig. 7, we calculated $d_{31 \text {,domain }}$ using the $\kappa$ values shown in Fig. 8 . The resultant $d_{31 \text { domain }}$ is plotted as a function of the applied field in Fig. 9. With zero stress, the calculated $d_{31 \text {,omain }}$ was about $-250 \times 10^{-12} \mathrm{~m} / \mathrm{V}$ at $E=400$ $\mathrm{kV} / \mathrm{m}$, which is comparable to the $d_{31}$ value measured from the resonance experiments. It should be noted that the resonance experiments were performed at low fields, and the values obtained from resonance measurements are a rough guide rather than precise values. Nevertheless, the reasonable agreement between the calculated $d_{31 \text {,domain }}$ and that from resonance measurements indicates that domain reorientation accounts for most of the $d_{31}$ coefficient in these EC-76 PZT materials, which is consistent with the finding in other soft PZT systems. ${ }^{22}$ Moreover, the transverse tensile stress showed a profound effect on the estimated $d_{31 \text {,domain }}$ at high electric fields. With no transverse tensile stress, $d_{31 \text {,domain }}$ became smaller at a high electric field, reflecting the saturation of $\kappa$ at higher electric fields. In contrast, with a transverse tensile stress, $d_{31 \text {,domain }}$ increased with an increasing electric field. At 75 $\mathrm{MPa}, d_{31 \text {, domain }}$ changed from $-250 \times 10^{-12} \mathrm{~m} / \mathrm{V}$ at $400 \mathrm{kV} / \mathrm{m}$ to $-350 \times 10^{-12} \mathrm{~m} / \mathrm{V}$ at $900 \mathrm{kV} / \mathrm{m}$, an increase of $\sim 40 \%$ in magnitude. Meanwhile, at $900 \mathrm{kV} / \mathrm{m}, d_{31 \text {,domain }}$ changed from $-200 \times 10^{-12} \mathrm{~m} / \mathrm{V}$ at zero stress to $-350 \times 10^{-12} \mathrm{~m} / \mathrm{V}$ at 75 $\mathrm{MPa}$, an increase of $75 \%$ in magnitude by the transverse tensile stress. These results support that the enhanced axial displacements in the Rainbow, Thunder, and PrinDrex actuators are in part caused by the enhanced piezoelectric coefficient by the transverse tensile stress present in these actuators. The enhanced $d_{31 \text {, domain }}$ value at high electric fields is a result of the enhanced domain reorientation at higher electric fields by the transverse tensile stress.

\section{Conclusions}

The effect of a transverse tensile stress on the electric-fieldinduced domain reorientation was investigated in situ by measuring the intensity ratio, $I_{(\mathrm{OO} 2)} / I_{(200)}$, of the tetragonal soft PZT in the presence of an electric field, as well as a transverse tensile stress using XRD. The in situ XRD experiments give quantitative information about the $90^{\circ}$-domain reorientation induced by an electric field in the presence of a transverse tensile stress in the soft PZT materials. We showed that a transverse tensile stress changed the electric-field dependence of $I_{(002)} / I_{(200)}$, especially at higher electric fields. Without a transverse tensile stress, $I_{(002)} / I_{(200)}$ began to saturate at $E=\sim 800 \mathrm{kV} / \mathrm{m}$. With a transverse tensile stress of $75 \mathrm{MPa}, I_{(\mathrm{OO} 2)} / I_{(200)}$ increased with an upward curvature with the electric field. This indicated that a transverse tensile stress enhanced the field-induced $90^{\circ}$-domain reorientation. With a simple model (Eq. (9)), the part of the piezoelectric coefficient 


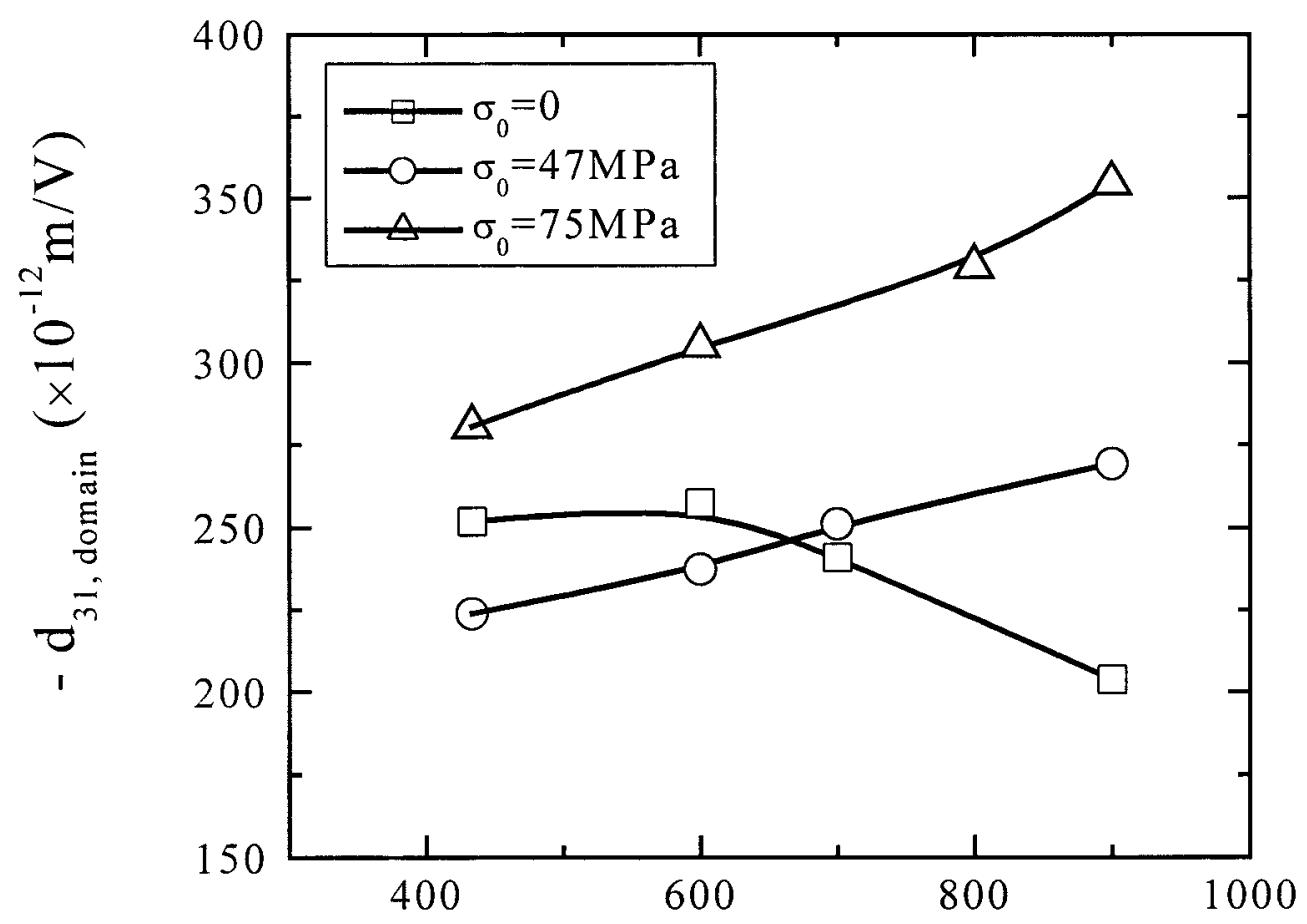

\section{Electric Field $(\mathrm{kV} / \mathrm{m})$}

Fig. 9. The deduced $d_{31 \text {,domain }}$ versus electric field at various tensile stresses where $d_{31 \text {,domain }}$ is the part of the $d_{31}$ that is caused by the $90^{\circ}$-domain reorientation.

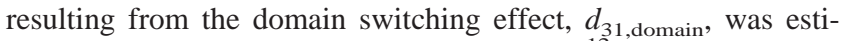
mated. The estimated $d_{31 \text {,domain }}=250 \times 10^{-12} \mathrm{~m} / \mathrm{V}$ at $400 \mathrm{kV} / \mathrm{m}$ was comparable to the $d_{31}$ value obtained using the resonance method at low electric fields. This indicated that domain switching accounted for most of the $d_{31}$ coefficient in these soft PZT materials. At zero stress, the $d_{31 \text {, domain }}$ decreased with an increasing electric field from $-250 \times 10^{-12} \mathrm{~m} / \mathrm{V}$ at $400 \mathrm{kV} / \mathrm{m}$ to about $-200 \times 10^{-12} \mathrm{~m} / \mathrm{V}$ at $900 \mathrm{kV} / \mathrm{m}$, because of the saturation in domain reorientation. In contrast, when the sample was subject to a transverse tensile stress, $d_{31 \text {,domain }}$ increased with an increasing electric field. At $75 \mathrm{MPa}, d_{31 \text {, domain }}$ increased from $-250 \times 10^{-12}$ $\mathrm{m} / \mathrm{V}$ at $400 \mathrm{kV} / \mathrm{m}$ to about $-350 \times 10^{-12} \mathrm{~m} / \mathrm{V}$ at $900 \mathrm{kV} / \mathrm{m}$, an increase of $\sim 40 \%$. Comparison of $d_{31 \text {,domain }}$ at a fixed electric field showed an even higher increase in the $d_{31 \text {,domain }}$ by the transverse tensile stress. At $900 \mathrm{kV} / \mathrm{m}, d_{31 \text {,domain }}$ increased from about $-200 \times 10^{-12} \mathrm{~m} / \mathrm{V}$ at zero stress to about $-350 \times 10^{-12}$ $\mathrm{m} / \mathrm{V}$ at $75 \mathrm{MPa}$, an increase of $75 \%$ by the transverse tensile stress. The enhanced $d_{31 \text {,domain }}$ at higher electric fields in the presence of a transverse tensile stress was caused by the enhanced domain reorientation at higher electric fields by the transverse tensile stress.

\section{References}

${ }^{1}$ J. M. Herbert, Ferroelectric Transducers and Sensors, Gordon and Breach, New York, 1982.

${ }^{2}$ S.-E. Park and T. R. Shrout, "Ultrahigh Strain and Piezoelectric Behavior in Relaxor Based Ferroelectric Single Crystals,” J. Appl. Phys., 82 [4] 1804-11 (1997).

${ }^{3}$ Q.-M. Wang and L. E. Cross, "Piezoelectric Pseudoshear Multilayer Actuator," Appl. Phys. Lett., 72 [18] 2238-40 (1998).

${ }^{4}$ V. Mueller and Q. M. Zhang, "Nonlinear and Scaling Behavior in Donor-Doped Lead Zirconate Titanate Piezoceramic," Appl. Phys. Lett., 72 [21] $2692-94$ (1998).

${ }^{5}$ V. D. Kugel and L. E. Cross, "Behavior of Soft Piezoelectric Ceramics under High Sinusoidal Electric Fields," J. Appl. Phys., 84 [5] 2815-30 (1998).

${ }^{6}$ Q.-M. Wang, Q. Zhang, B. Xu, R. Liu, and L. E. Cross, "Nonlinear Piezoelectric Behavior of Ceramic Bending Mode Actuators under Strong Electric Fields," J. Appl. Phys., 86 [6] 3352-60 (1999).
${ }^{7}$ G. H. Haertling, "RAINBOW Ceramics-A New Type of Ultra-HighDisplacement Actuator,” Am. Ceram. Soc. Bull., 73 [1] 93-96 (1994).

${ }^{8}$ Y. Sugawara, K. Onitsuka, S. Yoshikawa, Q. Xu, R. E. Newnham, and K. Uchino, "Metal-Ceramic Composite Actuators," J. Am. Ceram. Soc., 75 [4] 996-98 (1992).

${ }^{9}$ S. A. Wise, "Displacement Properties of Rainbow and Thunder Piezoelectric Actuators," Sens. Actuators, 69, 33-38 (1998).

${ }^{10}$ J. S. Vartuli, D. L. Milius, X. Li, W.-H. Shih, W. Y. Shih, R. K. Prud'homme, and I. A. Aksay, "Processing of a High-Displacement Ceramic-Ceramic Flextentional Actuator"; to be submitted to J. Am. Ceram. Soc.

${ }^{11}$ X. Li, J. S. Vartuli, D. L. Milius, I. A. Aksay, W. Y. Shih, and W.-H. Shih, "Electromechanical Properties of a Ceramic $d_{31}$-Gradient Flextensional Actuator," J. Am. Ceram. Soc., 84 [5] 996-1003 (2001).

${ }^{12} \mathrm{G}$. Li, E. Furman, and G. H. Haertling, "Stress-Enhanced Displacement in the PLZT Rainbow Actuators," J. Am. Ceram. Soc., 80 [6] 1382-88 (1997).

${ }^{13}$ (a)R. G. Bryant, "Process for Preparing a Tough, Soluble, Aromatic, Thermoplastic Copolyimide," U.S. Pat. No. 5639 850, June 17, 1997. (b)R. F. Hellbaum, R. G. Bryant, and R. L. Fox, "Thin Layer Composite Unimorph Ferroelectric Driver and Sensor," U.S. Pat. No. 5632 841, May 27, 1997.

${ }^{14}$ R. Y. Nishi, "Effect of One-Dimensional Pressure on the Piezoelectric Properties of Several Transducer Ceramics," J. Acoust. Soc., 40 [2] 486-95 (1966).

${ }^{15}$ H. H. A. Krurger, "Stress Sensitivity of Piezoelectric Ceramics: Part 3. Sensitivity to Compressive Stress Perpendicular to the Polar Axis," J. Acoust. Soc., 43 [3] 636-45 (1968).

${ }^{16} \mathrm{H}$. Cao and A. G. Evans, "Nonlinear Deformation of Ferroelectric Ceramics," J. Am. Ceram. Soc., 76 [4] 890-96 (1993).

${ }^{17}$ A. B. Shaufer, "Ferroelastic Properties of Lead Zirconate Titanate Ceramics," J. Am. Ceram. Soc., 79 [10] 2631-40 (1996).

${ }^{18}$ E. C. Subbarao, M. C. McQuarrie, and W. R. Ruessem, "Domain Effects in Polycrystalline Barium Titanate," J. Appl. Phys., 28, 1194-1200 (1957).

${ }^{19}$ M. Hammer, C. Monty, A. Endriss, and M. J. Hoffmann, "Correlation between Surface Texture and Chemical Composition in Undoped, Hard, and Soft Piezoelectric PZT Ceramics," J. Am. Ceram. Soc., 81 [3] 721-24 (1998).

${ }^{20}$ D. Berlincourt, D. R. Curran, and H. Jaffe, Physical Acoustics, Vol. 1A; pp. 169-269. Edited by W. P. Mason. Academic Press, New York, 1964.

${ }^{21}$ T. Tsurumi, Y. Kumano, N. Ohashi, T. Takenaka, and O. Fukunaga, " $90^{\circ}$ Domain Reorientation and Electric-Field Induced Strain of Tetragonal Lead Zirconate Titanate Ceramics, Part I," Jpn. J. Appl. Phys., 36, 5970-75 (1997).

${ }^{22}$ Q. M. Zhang, H. Wang, N. Kim, and L. E. Cross, "Direct Evaluation of Domain-Wall and Intrinsic Contributions to the Dielectric and Piezoelectric Response and Their Temperature Dependence on the Lead Zirconate-Titanate Ceramics," J. Appl. Phys., 75, 454 (1994). 